\title{
Using the Mercator ocean forecasting system to compute coastal maritime pollution risk indicators on the Atlantic European coasts
}

\author{
S. Besnard ${ }^{1}$, E. Dombrowsky ${ }^{1} \&$ P. Bahurel $^{2}$ \\ ${ }^{I}$ Space Oceanography Division, CLS, Toulouse, France \\ ${ }^{2}$ Mercator Ocean, Toulouse, France
}

\begin{abstract}
Mercator, the French oceanic forecast centre, provides in real-time a full 3D forecast of the ocean dynamics. Synthesizing such complex information into simple indicators useful for decision makers is a challenge in the context of GMES. We have implemented simple pollution indicators based on a semi lagrangian particle drift simulation using the Mercator surface current forecast and ECMWF wind speed. Shipping routes are the main places for genesis of oil spills. We have simulated the drift of particles sown daily along the main ship routes defined from the traffic separation schemes in the North Atlantic for a 3year period. For each season, we count the number of particles run aground by area of one degree per one degree. According to this number we allocate a pollution risk level to the section of the coast corresponding to the grounding. We then produce the first indicator which is a map showing the pollution risk level along the shore with a traffic light color scale: green, yellow, red, with red meaning high risk. The second indicator is deduced from the first one. Particles are tracked back to their initial location to identify the potential origin of the coastal pollution. This shows sections of ship routes with potential coastal pollution risk in the case of pollutant sewage along the ship route. The robustness of the computation of these indicators was assessed using the surface current from several other GODAE operational assimilation centers such as FOAM and RSMAS-HYCOM to compute the same indicators. We have also added noise to Mercator currents to evaluate the dispersion of the results. We found that even if there are some local differences in the indicators, some results are robust; specifically we show that there are seasonal variations.
\end{abstract}

Keywords: maritime coastal pollution, indicator, drift model, Mercator, operational system, ship routes, black tide, hydrocarbon pollutant. 


\section{Introduction}

In the context of GMES (Global Monitoring for Environment and Security) and MERSEA (Maritime EnviRonment and Security for the European Area) projects, the European Union desires to set up synthetic indicators for decision makers and environment agencies. These indicators are supposed to improve management of environmental risks. Nowadays, one of the major risks of coastal pollution is hydrocarbon and oil pollution linked to the maritime transport. Black tides have disastrous ecological and economics repercussions for communities and species. Maritime transport improves continuously, so tankers accidents and illegal oil emptying represents significant potential risks of pollution.

Mercator, the French oceanic forecast centre, provides in real-time a full 3D forecast of the ocean dynamics since 2001. Data produced by Mercator is rich and complex. Consequently, to provide simple indicators to address a specific problem is thus a necessity.

By using a drift model, we simulated pollution due to the maritime transport during three years. Two indicators were created. The first one gives the coasts exposed to the maritime traffic pollution. The second indicator shows parts of the ships route that lead to significant coastal maritime pollution.

In this paper, we first study characteristics of the drift model and how ship routes were integrated in the simulation. Secondly, the creation of the two indicators is assessed. Finally, we see the robustness tests which justify indicators existence.

\section{Experimental protocol}

\subsection{Drift model creation}

We have implemented a semi lagrangian particles drift model using a linear combination of Mercator surface currents and ECMWF wind speed for the advection (Hernandez [1]).

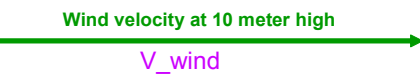

Total velocity at the

boundary ocean atmosphere

$\approx \alpha \% V_{\_}$curr $+\beta \% V_{\_}$wind

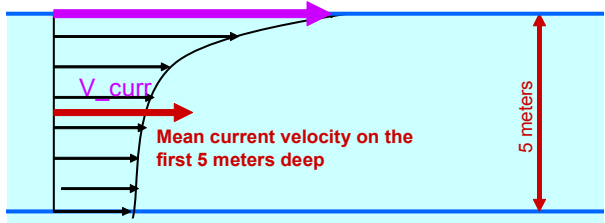

Figure 1: Combination of current and wind velocities. 
Moreover, even if wind speed is already contained in the Mercator surface currents, we need to intensify it. In fact, Mercator surface currents are those of the first five meters deep which are not representative of the surface currents due to the decrease of its intensity with the depth (fig. 1). Although this method is simple, it is efficient for a wind speed percentage included between 2 and 3\% (Hontarrède [2]).

The surface currents are from PSY2v1, the North Atlantic $1 / 15^{\circ}$ resolution prototype, re-interpolated on a $1 / 6^{\circ}$ resolution grid called Vitrine Data.

\subsection{Model validation}

The drift model was validated on the period of the Prestige tanker sinking in 2002. Figure 2 a shows the observations of the black tide evolution during the twelve weeks after Prestige sinking. On figure $2 b$, we observe about one hundred particles put where the sinking took place and drifting on the same twelve-week period. The percentage of wind speed use for the advection is $3 \%$. We can see a good time and space concordance between the observations and the model and so we consider that the drift model is validated.

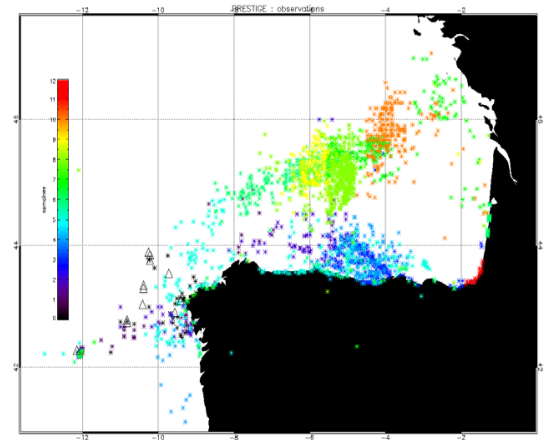

(a)

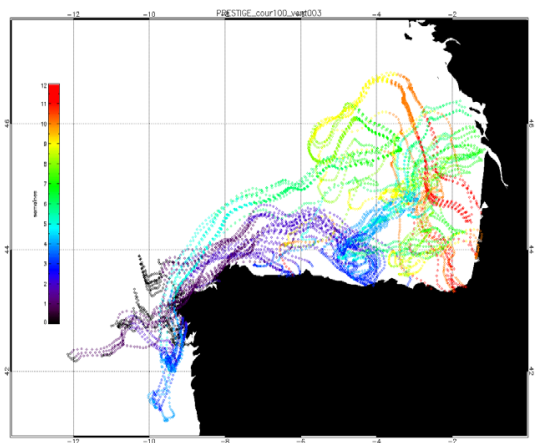

(b)

Figure 2: $\quad$ Comparison between observations and model.

\subsection{Shipping routes}

One hypothesis that we have made is that shipping routes are the main places for genesis of oil spills. As the tanker boats are most of the time responsible for black tides, it seems a quite good hypothesis.

From the European Atlantic traffic separation schemes (stars on figure 3) established by the IMO (International Maritime Organisation), we set up shipping routes. Traffic separation schemes serve to the traffic regulation for boats identity checks. Generally, they are located near capes and straits. 


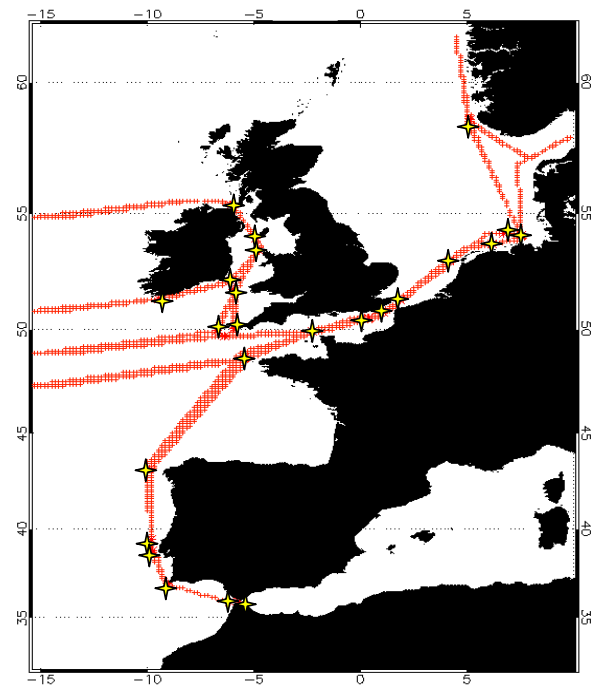

Figure 3: $\quad$ Ship routes defined from traffic separation schemes.

\section{Coastal maritime pollution risk indicators}

\subsection{Scenario of simulation}

We have simulated the drift of particles sown daily along main ship routes defined from the traffic separation schemes for a 3-year period. Each particle drift during a period of six month maximum, but most of the time it runs aground before. Globally, this represents about 1500 particles per day and 1.6 million particles for the entire simulation, enough to elaborate a statistical approach.

\subsection{The first indicator: black tides risks on the coasts}

At the end of the simulation, we count the number of particles run aground by area of one degree per one degree for each season. According to this number we allocate a pollution risk level to the section of the coast corresponding to the grounding. We then produce the first indicator which is a map showing the pollution risk level along the shore with a traffic light color scale: green, yellow, red, red meaning high risk (fig. 4a). For instance, Brittany is a coast expounded to a risk of maritime pollution.

\subsection{The second indicator: shipping routes parts leads to a coastal maritime pollution}

The second indicator is deduced from the first one. Particles are tracked back to their initial location to identify the coastal pollution potential origin. Therefore, we produce a map showing particles on their initial position with their final colour (fig. 4b). This shows sections of ship routes with potential coastal 
pollution risk in the case of pollutant sewage along the ship route. Typically, if an accident takes place on high risk area shipping routes, the coastal maritime pollution will be important and spread along a big part of the littoral. For instance, pollution risk in Brittany comes from shipping routes near Ouessant traffic separation scheme.

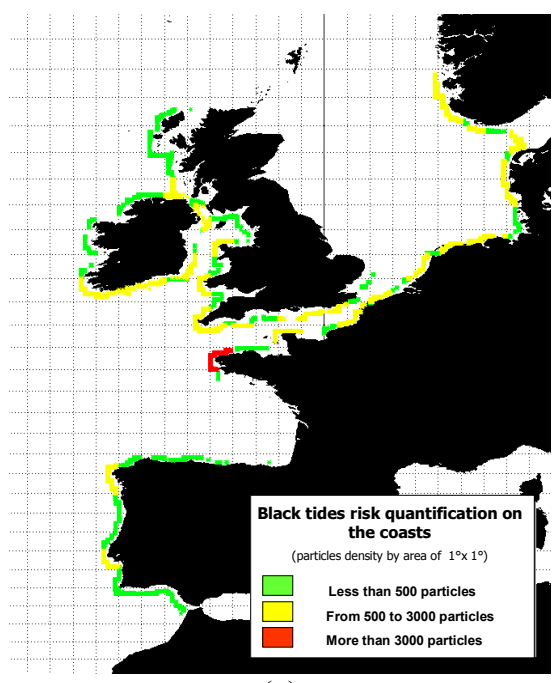

(a)

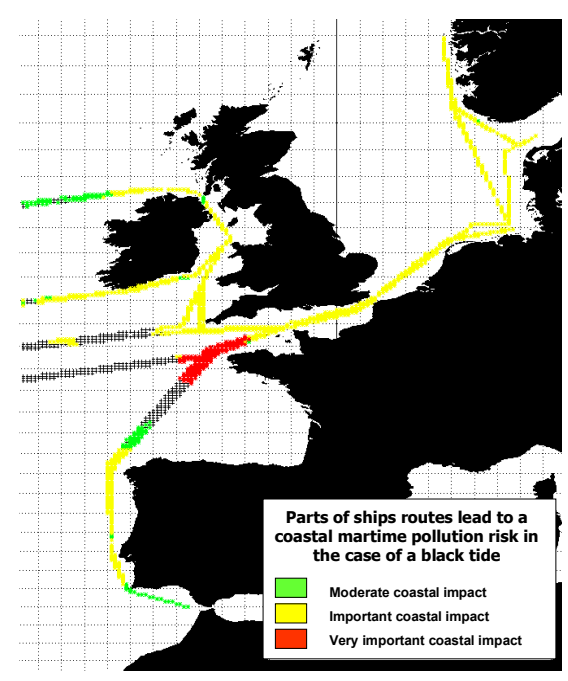

(b)

Figure 4: Maritime coastal pollution indicators.

\subsection{Results of the 3-year simulation}

From this 3-year simulation, it seems possible to discern a seasonal signal. High risks of pollution are more important in winter; this is correlated to the increase of wind during this period.

To assess the interest and the accuracy of these indicators, several robustness tests were realized.

\section{Indicators robustness}

\subsection{The different tests}

The robustness of these indicators was assessed by different ways (tab. 1).

The first one was by using surface currents coming from several other GODAE (Global Ocean Data Assimilation Experiment) operational assimilation systems such as FOAM (English model), RSMAS-HYCOM (American model) to compute the same indicators. Data from MERCATOR, FOAM and HYCOM models were recovered on the MERSEA website. The advantage of this method is that all data were set on a same grid. 
Another kind of test was to add noise to Mercator currents to evaluate the dispersion of the results. We employed an ensemble approach with the Monte Carlo method. In these two cases, to simplify simulations we used only surface currents without adding the wind speed component.

These cases were complemented by tests about the influence of the wind speed percentage for particles advection.

Table 1: $\quad$ Indicators robustness tests.

\begin{tabular}{|c|c|c|c|c|}
\hline $\mathbf{n}$ & Model data & Curr. & Wind & Type grid \\
\hline \multicolumn{5}{|c|}{ DRIFTING WITHOUT WIND } \\
\hline 1 & Mercator Vitrine PSY2v1 & $100 \%$ & $0 \%$ & $\mathbf{1} / \mathbf{6}^{\circ}$ mercator projection \\
\hline 2 & Mercator MERSEA & $100 \%$ & $0 \%$ & $1 / 6^{\circ}$ regular \\
\hline 3 & Hycom MERSEA & $100 \%$ & $0 \%$ & $1 / 6^{\circ}$ regular \\
\hline 4 & Foam MERSEA & $100 \%$ & $0 \%$ & $1 / 6^{\circ}$ regular \\
\hline 5 & Mercator Vitrine PSY2v1 Perturbed & $100 \%$ & $0 \%$ & $\mathbf{1} / \mathbf{6}^{\circ}$ mercator projection \\
\hline \multicolumn{5}{|c|}{ REAL DRIFTING (WITH WIND) } \\
\hline 6 & Mercator Vitrine PSY2v1 & $100 \%$ & $2 \%$ & $\mathbf{1} / \mathbf{6}^{\circ}$ mercator projection \\
\hline 7 & Mercator Vitrine PSY2v1 & $100 \%$ & $2.5 \%$ & $1 / 6^{\circ}$ mercator projection \\
\hline 8 & Mercator Vitrine PSY2v1 & $100 \%$ & $3 \%$ & $\mathbf{1 / 6 ^ { \circ }}$ mercator projection \\
\hline
\end{tabular}

Consequently, influence of the different model data is view with tests 2 to 4 . Grid influence is studied in tests 1 and 2. The Monte Carlo method is applied in the test 5 in comparison with the original test 1 . And we see the impact of different percentage of wind speed with tests 6 to 8 .

\subsection{Test 2 to 4: data sources influence}

Data stemming from the different ocean models (FOAM, HYCOM, MERCATOR) gives, locally, different indicators. Moreover, bathymetries used in each model are different. That's why a lot of particles have not the same grounding and this directly impacts the indicators. We tried to force particles grounding by using the same bathymetry, but to harmonize all the models we are forced to go away from the coasts and use a grounding criterion less severe which is not representative of the littoral cut. So, to have a better mean of comparison of the different oceanic systems, coastal data needs to be improved. However, indicators created from HYCOM and MERCATOR data presents some similarities for each season.

\subsection{Tests 1 and 2: grid influence}

Although, the grids employed in these two tests are not very different, because both have a $1 / 6^{\circ}$ step resolution, it's not the same kind of grid and consequently grid points are not the same. In this case, we conclude that data interpolation does not suffer from the repartition on the grid. But it will be interesting to make the same test with a bigger difference of grid resolution. 


\subsection{Tests 1 and 5: noise added influence}

By adding noise to current velocity used in the drift model, we try to show that indicators produced are not modified too much. To create a coherent noise with the original signal which is the current velocity, we use a structured noise (fig. 5) limited by a percentage of the standard deviation of each current velocity components, zonal and meridian.

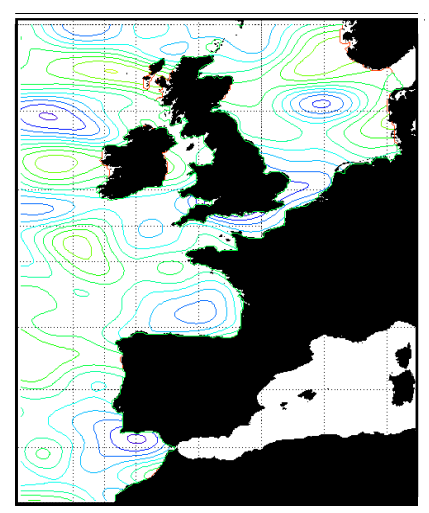

Figure 5: One structured noise grid.

To have a statistical approach, like the Monte Carlo method (Evensen [3]), we have created about two hundred structured noise grids, one hundred for each component. We add these noises to the current velocity and we make one hundred simulations during one season (not three years because it is too long and costly). Then we calculate the average of each particle final position and we compare to the particle position of the non-perturbed simulation (fig. 6). If the results are relatively similar, we obtain the same indicators and we conclude that their production is robust even if the input signal is perturbed.

We made this work for several percentages of noise, $\pm 1 \%, \pm 10 \%, \pm 20 \%$ and $\pm 50 \%$. Obviously, the more perturbed the signal is, the less similar indicators are.

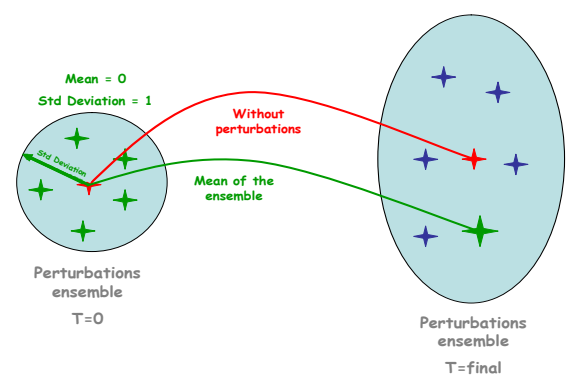

Figure 6: Monte Carlo ensemble method theory. 
It appears that perturbations up to $10 \%$ seem to be acceptable because indicators are not too much modified. Therefore, we consider the indicators making robusts. Beyond $10 \%$, perturbations are too important and modified the signal too much.

\subsection{Tests 6 to 8: wind speed variation influence}

To test the influence of the wind speed percentage applied on the particle advection, we made indicators with different percentage of wind velocity, $2 \%$ (figure 7a), 2.5\% and 3\% (figure 7b) which are the percentages the most applied for this type of drift advection. Results shows that the indicators produced are very similar. Thus, a wind perturbation does not have a lot of impact on the indicators production.

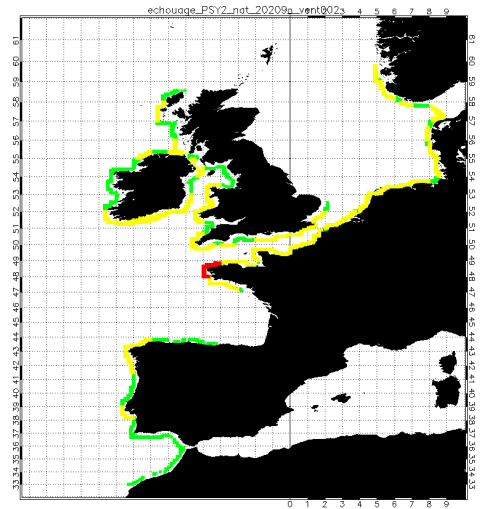

(a)

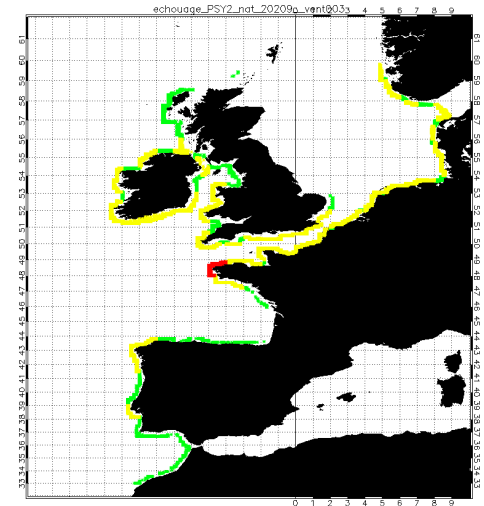

(b)

Figure 7: Wind speed influence on indicators production.

\section{Conclusion}

This work shows that it is possible to give clear information through two simple indicators that are dedicated to coastal maritime pollution.

The first indicator gives an estimate on the risk of coastal maritime pollution. The second indicator identifies the sections of the shipping route where the risk is critical for coastal maritime pollution.

The quality of the indicators produced is quite good. We have found that even if there are some local differences in the indicators due to the influence of the grid, the data source, the wind speed or the signal noise, some results are robust; especially we show that there are seasonal variations.

To produce those indicators on a monthly basis is feasible. It concerns the Atlantic and the Mediterranean Sea.

The interest of these indicators consists by the acknowledgement they bring on the maritime pollution risk in two strategic locations, where the genesis and the beaching take place. 


\section{Acknowledgements}

This work has been carried out thanks to a direct financing of Mercator Ocean and by CLS under contract $\mathrm{n}^{\circ} 04 / 1215183 / \mathrm{G}$ for Mercator Ocean.

\section{References}

[1] Hernandez, F., Comparaison et combinaison de données altimétriques et lagrangiennes. Application à la campagne SEMAPHORE-93, UMR39/GRGS/OMP, Université Paul Sabatier (Toulouse III), 276,1995.

[2] Hontarrède M., Pollution en mer, Observer et prévoir leur dérive. MET MAR n²00, Météo-France, sept. 2003.

[3] Evensen, G., Sequential data assimilation with a non-linear quasigeostrophic model using Monte Carlo methods to forecast error statistics. Journal of Geophysical Research 99 (C5): 10143-10162. 1994. 